\title{
Structural Characterization of Stress-Induced Martensitic Transformation in a Polycrystalline Austenitic Fe-Mn-Si-Cr Alloy
}

\author{
Shotaro Senoo ${ }^{1}$, Kozo Shinoda ${ }^{1}$, Masugu Sato ${ }^{2}$, Tadatsugu Maruyama ${ }^{3}$ and Shigeru Suzuki ${ }^{1, *}$ \\ ${ }^{1}$ Institute of Multidisciplinary Research for Advanced Materials, Tohoku University, Sendai 980-8577, Japan \\ ${ }^{2}$ JASRI/SPring-8, Sayo-gun, Hyogo 679-5198, Japan \\ ${ }^{3}$ Awaji Materia Co. Ltd., Tokyo 101-0052, Japan
}

Stress-induced martensitic transformation in Fe-Mn-Si alloys is characterized by the transformation of the fcc matrix to the hcp phase, which is generally reversible. In this study, Debye rings obtained by monochromated X-ray diffraction using synchrotron radiation were used for analyzing the structural change of the fcc matrix to the hcp phase in a polycrystalline austenitic Fe-Mn-Si-Cr alloy that was deformed by the tensile test at room temperature. Structural changes resulting from the reverse transformation due to heating were also studied. The results showed that the occurrence of the stress-induced martensitic transformation was not uniform, but depended on the relationship between the orientation of polycrystalline grains and the tensile direction. The transformation appears to preferentially occur in grains with large Schmid factors for the shear of $[\overline{2} 11](111)$ in the fcc matrix, and the formation of hcp phases also depends on the orientation of grains. The reverse transformation due to heating does not necessarily occur in the crystallographically reversible route. This indicates that irreversible deformation induced by dislocations during the tensile test restricts the reversible transformation of the alloy. [doi:10.2320/matertrans.MRA2008034]

(Received January 28, 2008; Accepted March 12, 2008; Published May 25, 2008)

Keywords: stress-induced martenstic transformation, X-ray diffraction, synchrotron radiation, polycrystalline iron-based alloys, shape memory

\section{Introduction}

Fe-Mn-Si austenitic alloys containing a large amount of manganese are known to exhibit the shape memory effect, which occurs as a consequence of the reverse transformation of martensite formed by stress-induced transformation. ${ }^{1-3)} \mathrm{It}$ is considered in the stress-induced martensitic transformation that the face centered cubic (fcc) structure phase (austenite) is transformed to a hexagonal closed packed (hcp) structure phase (martensite) during deformation at about room temperature, and the reverse transformation is induced by heating up to approximately $700 \mathrm{~K}$. A model was proposed to explain the transformation of the fcc to the hcp phase, as illustrated in Fig. 1. A few percentage of chromium was added to these alloys in order to improve the corrosion resistance of the Fe-Mn-Si based shape memory alloys. ${ }^{4}$ In addition, the precipitation of carbides or nitrides has been utilized for improving the recovery strain in polycrystalline $\mathrm{Fe}-\mathrm{Mn}-\mathrm{Si}$ alloys. ${ }^{5-10)}$ As these alloys comprise non-precious elements, they are required to be applied to practical structural and functional materials.

In order to clarify the deformation mechanism of $\mathrm{Fe}-\mathrm{Mn}-\mathrm{Si}$ shape memory alloys, their mechanical properties, ${ }^{11-13)}$ and microstructure relevant to the crystallographic orientation in the stress-induced transformation of the fcc matrix to the hcp martensite phase ${ }^{14-16)}$ were investigated in Fe-Mn-Si alloys. Although the recovery strain induced by heating is an important parameter for characterizing the shape memory effect, it is known that the recovery strain in polycrystalline $\mathrm{Fe}-\mathrm{Mn}-\mathrm{Si}$ alloys is lower than that in single-crystal $\mathrm{Fe}-\mathrm{Mn}-\mathrm{Si}$ alloys, in which the recovery strain depends on the stress direction. The crystallographic orientation between the fcc matrix and hcp martensite phase has been studied using transmission electron microscopy and scanning electron

*Corresponding author, E-mail: ssuzuki@tagen.tohoku.ac.jp microscopy. The results show a microscopic morphology of the martensite; however, the overall orientation changes in stress-induced transformation and the reverse transformation of the alloys are unclear.

Therefore, X-ray diffraction measurement is a better method for characterizing the overall structural changes in polycrystalline alloys. This is because polycrystalline alloys consist of a large number of grains; where, the crystallographic orientation distribution plays an important role in the shape memory characteristics. In this work, Debye rings were analyzed by X-ray diffraction using synchrotron radiation in order to clarify the relationship between the macroscopic structural changes of a Fe-Mn-Si alloy by deformation.

\section{Experimental}

Sheets of Fe-Mn-Si-Cr shape memory alloys of approximately $0.8 \mathrm{~mm}$ in thickness were prepared following a previously used method. ${ }^{4,17)}$ The chemical composition of the shape memory alloy was Fe-28 mass\%Mn- 6 mass $\%$ Si5 mass $\% \mathrm{Cr}$, which is simply referred to as Fe-Mn-Si hereafter. The sheets were annealed at $1027 \mathrm{~K}$ for $1800 \mathrm{~s}$ to obtain a recrystallized microstructure. The grain size of the alloys was approximately $30 \mu \mathrm{m}$. The sheets were cut into the small shape of samples for tensile test with a gauge size of $0.7 \times 2.0 \times 10 \mathrm{~mm}$, in which the tensile test direction was the rolling direction of the sheets. The alloy sheets did not reveal a strongly textured microstructure, as shown later. The unidirectional tensile test was carried out at room temperature at the strain rate of $1 \times 10^{-4} \mathrm{~s}^{-1}$. The recovery treatment was carried out by heating at $673 \mathrm{~K}$ for $600 \mathrm{~s}$, as the samples were sufficiently recovered by this heating condition.

X-ray diffraction measurements of the polycrystalline $\mathrm{Fe}-\mathrm{Mn}-\mathrm{Si}$ were performed using synchrotron radiation, in order to obtain structural information from a large number of 


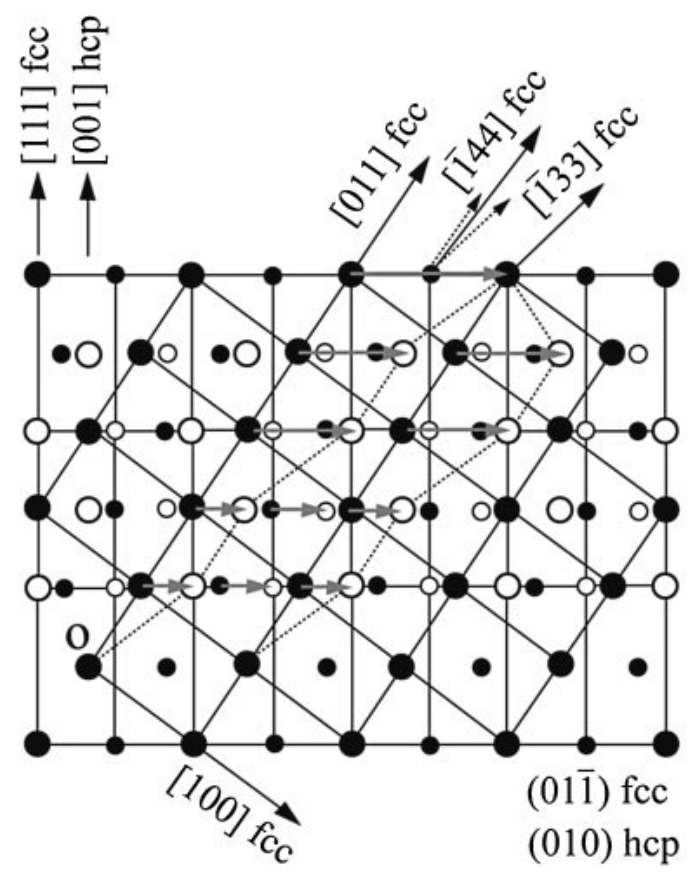

: fcc

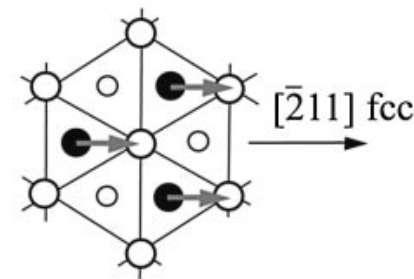

(111) fcc

(001) hcp

Fig. 1 Model showing crystallographic orientation relationship between fcc (solid mark) hcp (open mark) phases after the transformation induced by tensile deformation as observed from two different views (a) and (b).

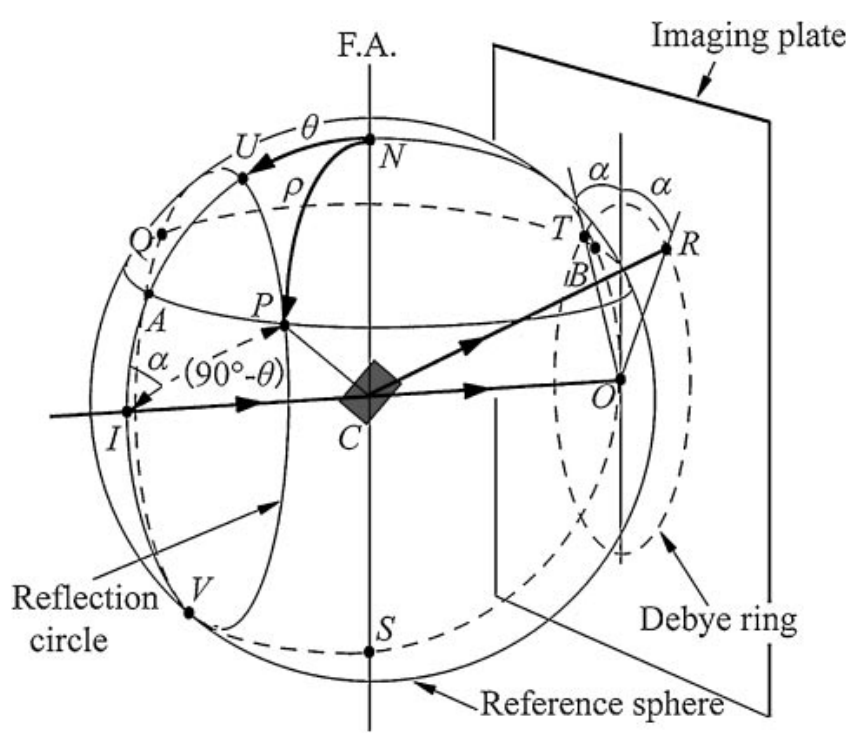

Fig. 2 Geometry of $(h k l)$ diffraction from a crystal having $[u v w]$ axis. FA is estimated from different diffractions.

grains. The beamline for the X-ray diffraction measurement was BL19B2 of SPring-8 (Hyogo, Japan), in which diffraction rings were measured using the Debye-Scherrer method. The photon energy during the measurements was $30 \mathrm{keV}$, with a wave length of $0.04127 \mathrm{~nm}$, and camera length of $210 \mathrm{~mm}$. Debye rings by X-ray diffraction using synchrotron radiation were recorded using an imaging plate. Diffraction intensity changes in Debye rings were used for analyzing structural changes in polycrystalline $\mathrm{Fe}-\mathrm{Mn}-\mathrm{Si}$ alloys by loading and heating.

The geometry of the diffraction measurements of a tensile sample is shown in Fig. 2, together with the orientation analysis procedure. ${ }^{18)}$ The crystallographic orientaion $[u v w]$ of the fiber axis (FA), that is consistent with the tensile stress axis, is estimated from changes of diffraction intensities by stress-induced transformation and reverse transformation. A sample with the $[u v w]$ axis oriented toward $N S$ is at $C$, which is normal to $I C$. $C P$ is the normal to a set of $(h k l)$ planes, at which diffraction occurs when they are inclined to the incident beam at an angle $\theta$ satisfying Bragg's law. Then, the $(h k l)$ pole should be on the circle $P U Q V$. The $(h k l)$ pole confined to the circle $P A Q B$ is the circle around the $[u v w]$ axis. Thus, diffraction of the $(h k l)$ pole occurs at $P$ and $Q$, which are intersections of $P U Q V$ and $P A Q B$, and the corresponding diffraction spots appear at $R$ and $T$ on the imaging plate. For the spherical triangle, $I P N$, the relationship between the angles $\rho, \theta$, and $\alpha$ is given as follows:

$$
\cos \rho=\cos \theta \cos \alpha
$$

By measuring $\alpha$ in the Debye rings on the imaging plate and calculating $\theta, \rho$ can be estimated. The index $[u v w]$ can be estimated from a set of values of $\rho$ measured for different $(h k l)$ poles.

\section{Results and Discussion}

\subsection{Structural changes in fcc matrix by tensile defor- mation and heating}

Figure 3(a) shows Debye rings for diffraction of recrystallized $\mathrm{Fe}-\mathrm{Mn}$-Si alloys. In order to compare the distribution of intensities of the diffracted X-ray, the Debye rings were three-dimensionally (3D) displayed as shown in Fig. 3(b). The results indicate that the recrystallized Fe-Mn-Si reveals the complete fcc austenitic structure in the polycrystalline sample.

The Debye rings and their 3D display for Fe-Mn-Si alloys deformed by $10 \%$ tensile deformation at room temperature are shown in Figs. 4(a) and (b), respectively. These results 
(a)

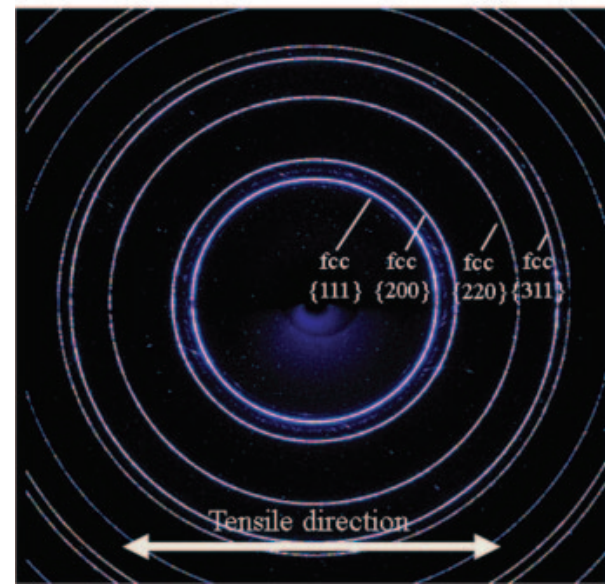

(b)

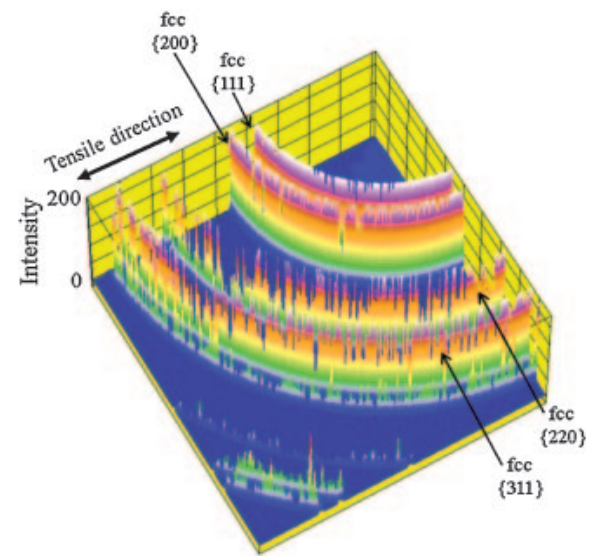

Fig. 3 (a) Debye rings and (b) their 3D display in (quasi-color) for the diffraction pattern from recrystallized Fe-Mn-Si alloys.

(a)

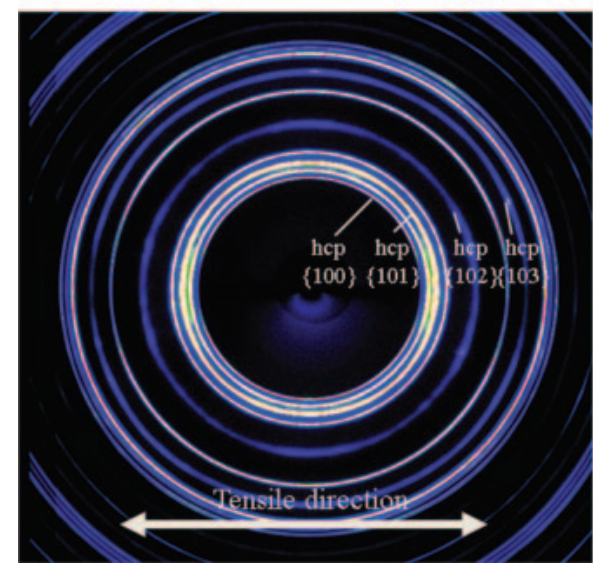

(b)

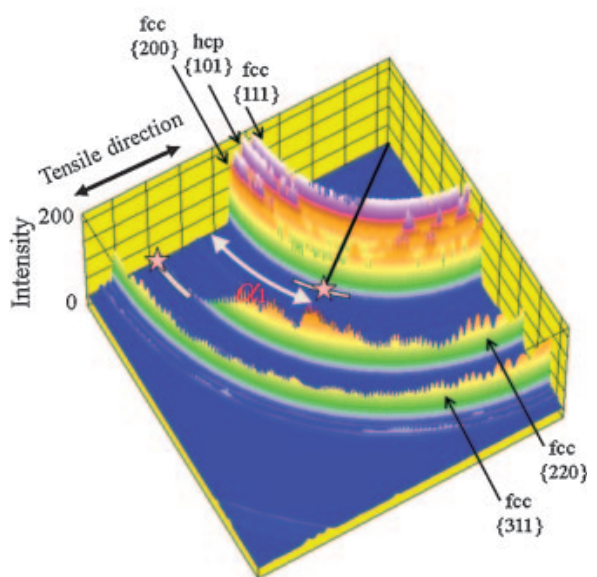

Fig. 4 (a) Debye rings and (b) their 3D display for the diffraction pattern from Fe-Mn-Si alloys deformed by $10 \%$ at room temperature.

(a)

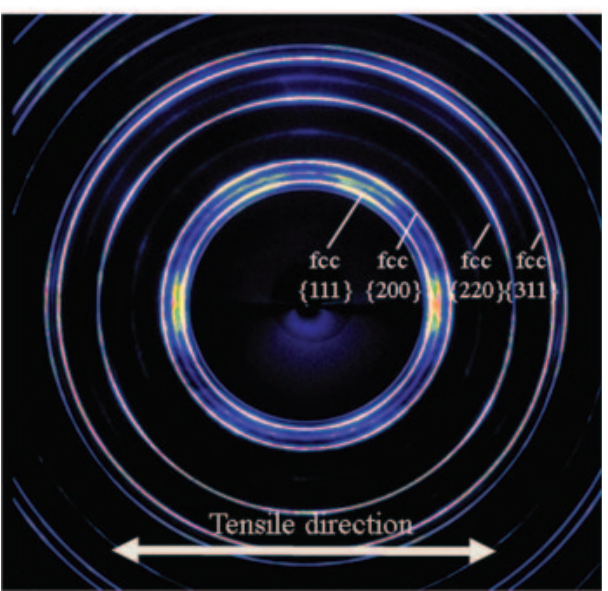

(b)

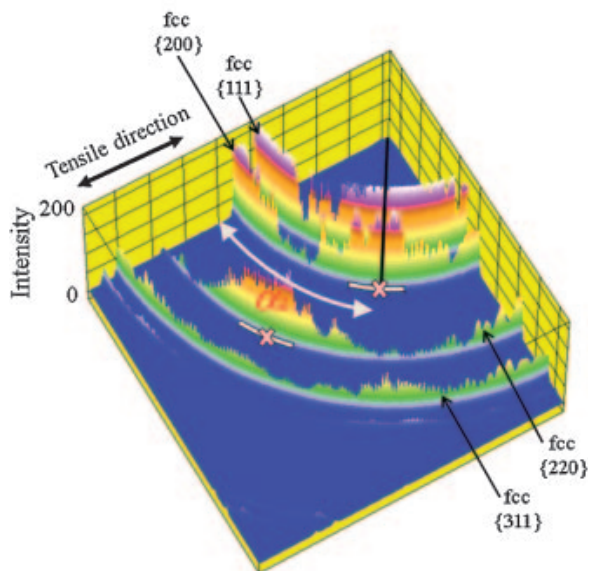

Fig. 5 Three-dimensional Debye rings of diffraction patterns from (a) Fe-Mn-Si alloys deformed by $10 \%$ at room temperature and (b) Fe-Mn-Si alloys heated to $673 \mathrm{~K}$ after $10 \%$ deformation. 
Table 1 Orientations of the fcc matrix whose diffraction intensities were preferentially decreased by stress-induced transformation.

\begin{tabular}{llll}
\hline Index & $\theta($ degree $)$ & $\alpha_{1}$ (degree) & $\rho$ (degree) \\
\hline 111 & 5.6 & 22 to 35 & 23 to 35 \\
\hline 200 & 6.5 & 30 to 50 & 28 to 47 \\
\hline 220 & 9.3 & -8 to 8 & 9 to 14 \\
\hline
\end{tabular}

Table 2 Orientations of the fcc matrix whose diffraction intensities were preferentially increased by reverse transformation.

\begin{tabular}{llcc}
\hline Index & $\theta($ degree $)$ & $\alpha_{1}$ (degree) & $\rho$ (degree) \\
\hline 111 & 5.6 & -12 to 12 & 6 to 10 \\
\hline 200 & 6.5 & 40 to 70 & 42 to 64 \\
\hline 220 & 9.3 & 23 to 39 & 18 to 36 \\
\hline
\end{tabular}

show that rings attributed to the hcp phase (martensite), which was formed by the stress-induced martensitic transformation, appeared during deformation. It should be noted that the $\{220\}$ diffraction intensities from the fcc matrix, which is oriented almost to the tensile direction, is decreased by the deformation. This indicates that the martensitic transformation occurred in grains with specific orientation in polycrystalline Fe-Mn-Si by tensile deformation. The hep phase also seems to be formed in grains with specific orientation. These orientation relationships relevant to the transformation will be discussed later. Parameters concerning the preferential orientation of fcc phase, which were transformed to hcp phase, can be determined from angles in these Debye rings by following the method shown in Fig. 2 . Table 1 summarizes the angles obtained from the distribution of the diffraction intensities from the fcc matrix, which were preferentially decreased by the stress-induced transformation.

Figures 5(a) and (b) show the Debye rings and their 3D display resulting from the diffraction of $\mathrm{Fe}-\mathrm{Mn}-\mathrm{Si}$ alloys heated at $673 \mathrm{~K}$ after the $10 \%$ tensile deformation. These Debye rings show that the diffraction intensities in the rings attributed to the hcp phase were decreased by heating, indicating that most hcp phases formed by the stress-induced martensitic transformation considerably disappeared by the recovery treatment. However, it is noted that some parts of the $\{220\}$ diffraction intensities from the fcc matrix were not perfectly recovered. This means that the reverse transformation did not fully occur under the present condition, while the stress-induced martensitic transformation preferentially takes please in polycrystalline $\mathrm{Fe}-\mathrm{Mn}$-Si by unidirectional deformation. Parameters on the preferential orientation of the fcc phase, which were recovered from the hcp phase, can be measured from angles in these Debye rings. The angles pertaining to the changes in diffraction intensities from the fcc matrix are listed in Table 2.

\subsection{Structural changes in hcp phase by tensile deforma- tion and heating}

In order to observe the diffraction intensities due to the hcp phase in the Debye rings, they were displayed in different views. Figures 6(a) and (b) show 3D Debye rings for Fe-Mn-
Si alloys deformed by $10 \%$ and subsequently heated to $673 \mathrm{~K}$, respectively. The results clearly show that the intensities of hcp $\{100\}$ rings were increased in specific orientations by the deformation. This indicates that the hcp phase was not uniformly formed, but formed preferentially in grains with a specific orientation in the polycrystalline Fe-Mn-Si by unidirectional deformation. On the other hand, the diffraction intensities assigned to the hcp phase formed by the tensile deformation almost disappeared on heating. This corresponds to the hcp-fcc reverse transformation following the stressinduced martensitic transformation.

Figures 7(a) and (b) display the 3D Debye rings in a different view for Fe-Mn-Si alloys deformed by $10 \%$ and those subsequently heated to $673 \mathrm{~K}$, respectively. The hcp $\{102\}$ and $\{103\}$ rings are significantly focused upon in this view. The results also show that the hcp phase formed by the deformation almost disappeared on heating, also indicating that the stress-induced martensitic transformation preferentially occurs in grains with specific orientations in polycrystalline $\mathrm{Fe}-\mathrm{Mn}-\mathrm{Si}$. As angles concerning the preferential formation of hcp phase are listed in Table 3, they will be compared with the results obtained from the fcc phase in the next section.

\subsection{Preferential crystallographic orientation of grains for transformation of polycrystalline Fe-Mn-Si alloys}

From the angles given in Tables 1 and 2 where the X-ray diffraction intensities were changed, the orientation of the stress axis inducing stress-induced transformation and reverse transformation can be estimated. Figure 8 shows a stereographic projection showing the orientation of the preferentially transformed fcc grains the stress axis by tensile deformation that was estimated from angles due to the intensity changes in different diffraction planes. The stereographic projection is displayed in the form [ $\overline{2} 11](111)$, which represent the shear direction and plane in Fig. 1, respectively. This result indicates that the orientations of grains that are preferentially transformed in the polycrystalline fcc matrix by tensile deformation are nearly around $\langle 144\rangle$. This appears to be in agreement with the model, in which grains oriented nearly to $\langle 144\rangle$ has a large Schmid factor for transformation of the fcc to hep phase, as shown in Fig. 1. Thus, authors maintain the view from the present results that the fcc - hcp transformation preferentially occurs in grains oriented to nearly $\langle 144\rangle$ by unidirectional tensile deformation, by which the diffraction intensities assigned to the fcc matrix were decreased.

On the other hand, a stereographic projection revealing the preferential orientation of fcc grains reversely transformed by heating is shown in Fig. 9. In this case, the preferential orientation of grains reversely transformed in the polycrystalline fcc matrix by heating is slightly shifted to [ 111$]$ direction from [144], although the orientation has some distribution. The irreversibility in the structural change by the stress-induced transformation and reverse transformation corresponds to the results on the irreversibility of the Debye rings as shown in Fig. 5. These results imply that the reverse hcp-fcc transformation is not perfect, and it may be attributed to irreversible slip deformation by dislocation motion. 
(a)

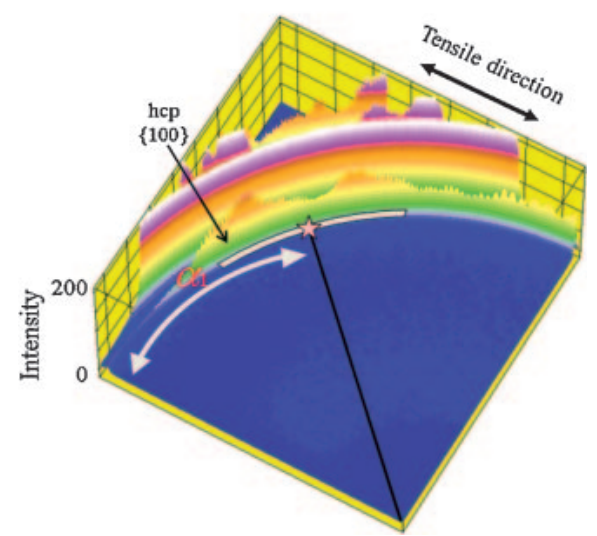

(b)

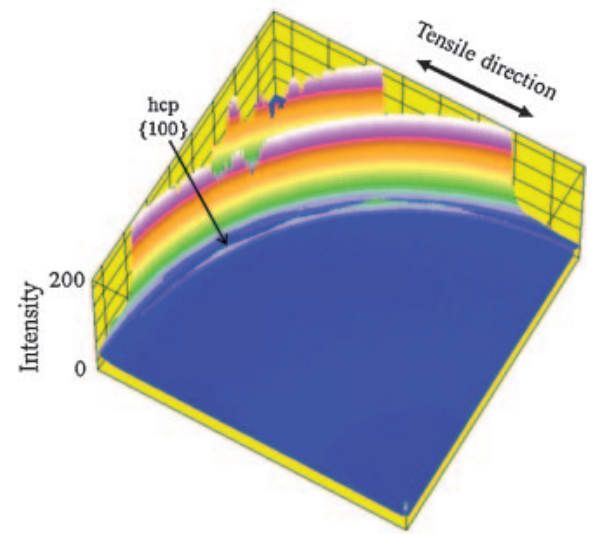

Fig. 6 Three-dimensional Debye rings of diffraction patters from (a) Fe-Mn-Si alloys deformed by $10 \%$ at room temperature and (b) Fe-Mn-Si alloys heated to $673 \mathrm{~K}$; hcp $\{101\}$ is also shown.

(a)

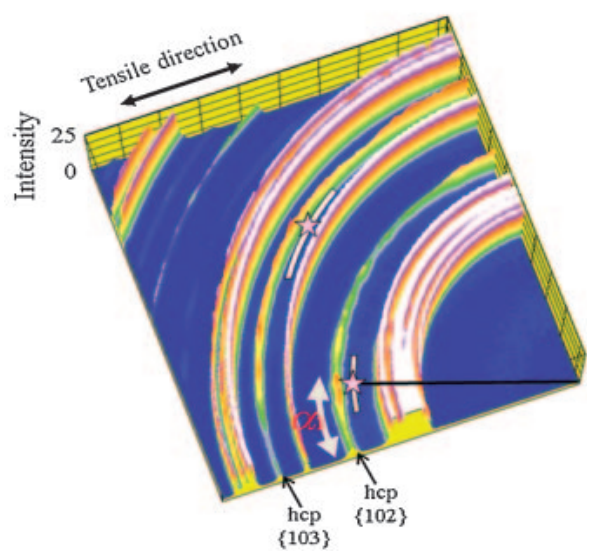

(b)

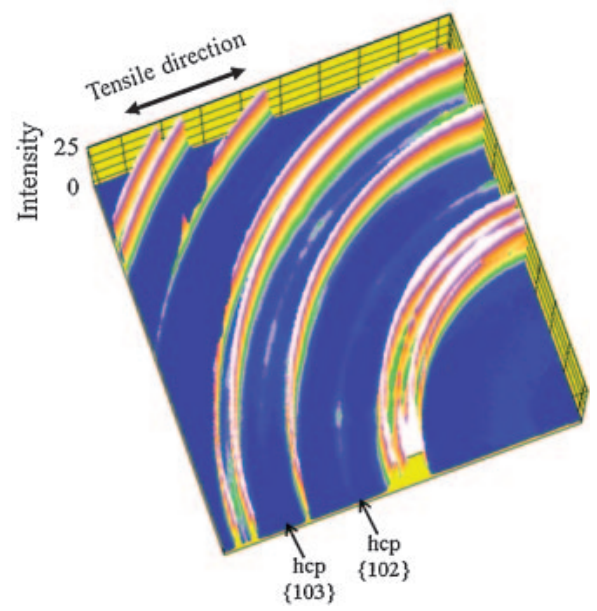

Fig. 7 3D Debye rings of diffraction patters from (a) Fe-Mn-Si deformed by $10 \%$ at room temperature and (b) Fe-Mn-Si heated at $673 \mathrm{~K}$ after $10 \%$ deformation, in which hcp $\{102\}$ and $\{103\}$ are shown.

Table 3 Orientations of the hcp phase whose diffraction intensities were preferentially increased by stress-induced transformation.

\begin{tabular}{cccc}
\hline Index & $\theta($ degree $)$ & $\alpha_{1}$ (degree) & $\rho$ (degree) \\
\hline 100 & 5.4 & 32 to 45 & 26 to 38 \\
\hline 101 & 6.1 & -8 to 8 & 6 to 10 \\
\hline 102 & 7.9 & 12 to 28 & 13 to 29 \\
& & & 80 to 90 \\
\hline 103 & 10.2 & 42 to 62 & 43 to 62 \\
& & & 80 to 90 \\
\hline
\end{tabular}

In addition, Figure 10 shows a stereographic projection revealing the orientation of the hcp phase preferentially formed by tensile deformation. The stereographic projection is displayed in the form [120](001\}, as referred in Fig. 1. This result indicates that the preferential orientation of the hcp phases transformed by tensile deformation is slightly shifted and dispersed to [120] direction. This suggests that the preferential orientation of the hcp phase formed from the fcc matrix is deviated from the ideal fcc-hcp orientation

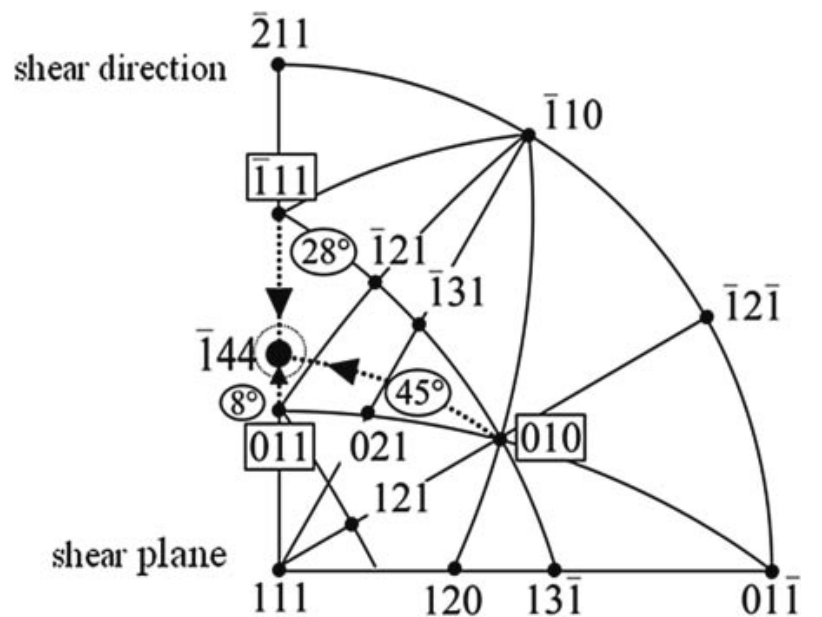

Fig. 8 Stereographic projection showing orientation of fcc grains preferentially transformed by tensile deformation. Shear plane and shear direction for stress-induced martensitic transformation are shown in [ $\overline{2} 11]$ and (111), respectively. Representative angles between the preferential orientation and the diffraction planes are denoted in the projection. The $\langle 144\rangle$ orientation with a large Schmid factor is shown for fcc-hcp transformation. 


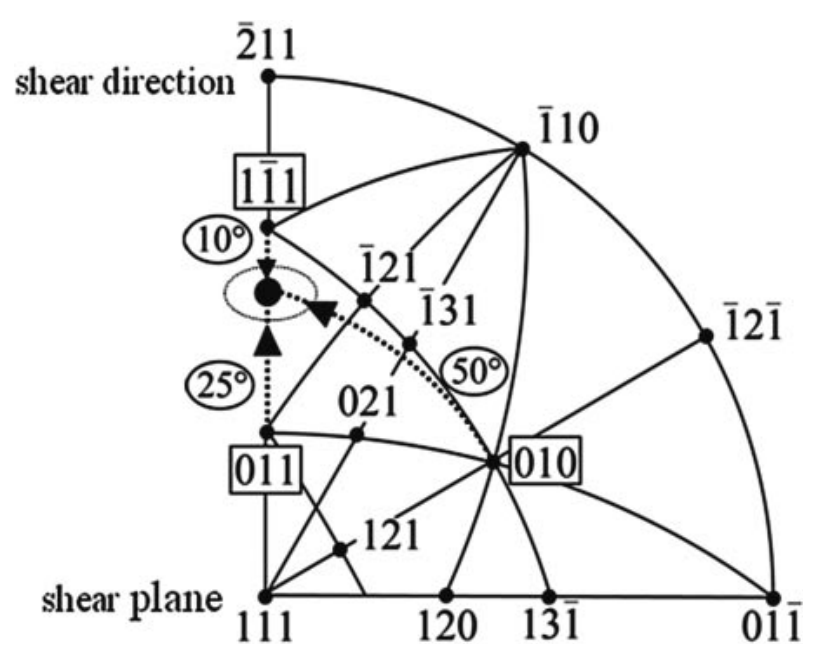

Fig. 9 Stereographic projection showing orientation of fcc grains preferentially reverse transformed by heating. Representative angles between the preferential orientation and the diffraction planes are denoted in the projection.

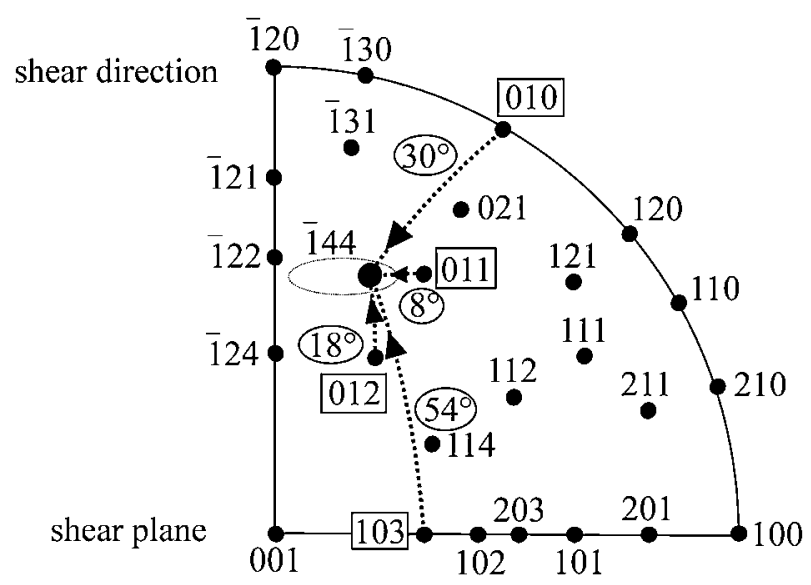

Fig. 10 Stereographic projection showing orientation of hcp grains preferentially formed by tensile deformation. Representative angles between the preferential orientation and the diffraction planes are denoted in the projection.

relationship; this may also be attributed to irreversible slip deformation due to dislocations.

Thus, it was inferred in the above analysis that grains with large Schmid factors for the fcc-hcp martensitic transformation are preferentially transformed in polycrystalline grains by unidirectional stress. Although the flow stress for stressinduced martensitic transformation may be slightly lower than that for slip deformation by dislocations, irreversible deformation due to dislocations may also occur during the overall stress-induced transformation. Such irreversible deformation may reduce the degree of the reverse transformation that contributes to the shape memory effect of $\mathrm{Fe}-\mathrm{Mn}-\mathrm{Si}$. As the present results imply that the preferential orientation of the fcc matrix is important in obtaining high shape memory characteristics, the texture in Fe-Mn-Si needs to be controlled to improve the shape memory characteristics as seen in iron based alloys. ${ }^{19,20)}$

\section{Conclusions}

The X-ray diffraction method using synchrotron radiation was used for characterizing the phase transformation in a Fe$\mathrm{Mn}-\mathrm{Si}$ shape memory alloy. Debye rings obtained by X-ray diffraction showed that the fcc matrix was partially transformed to an hcp martensite phase in this alloy by tensile deformation and reverse transformation of the hcp phase to the fcc matrix occurs by heating. The occurrence of stressinduced martensitic transformation depended on the relationship between the orientation of polycrystalline grains and the tensile direction; in particular, the transformation appears to preferentially occur in grains with large Schmid factors for the shear of $[\overline{2} 11](111)$ in the fcc matrix. The reverse transformation was not perfect, but it may be attributed to irreversible deformation due to dislocations. The formation of the hcp phase was in agreement with the results obtained for the fcc matrix.

\section{Acknowledgment}

The authors would like to express their sincere gratitude to Prof. Y. Waseda for his support to this work. This study is partially supported by Nippon Steel Corporation.

\section{REFERENCES}

1) A. Sato, E. Chishima, K. Soma and T. Mori: Acta Metall. 30 (1982) 1177-1183.

2) A. Sato, Y. Yamaji and T. Mori: Acta Metall. 34 (1986) 287-294.

3) A. Sato, E. Chishima, Y. Yajima and T. Mori: Acta Metall. 32 (1984) 539-574.

4) H. Ohtsuka, H. Yamada, T. Maruyama, H. Tanahashi, S. Matsuda and M. Murakami: ISIJ Inter. 30 (1990) 674-679.

5) S. Kajiwara, T. Kikuchi and N. Shinya: Scripta Mater. 44 (2001) 2809-2814.

6) T. Sawaguchi, T. Kikuchi, K. Ogawa, S. Kajiwara, Y. Ikeno, M. Kojima and T. Ogawa: Mater. Trans. 47 (2006) 580-583.

7) K. Ogawa, T. Sawaguchi, T. Kikuchi and S. Kajiwara: Mater. Trans. 48 (2007) 869-877.

8) A. Sato, H. Kubo and T. Maruyama: Mater. Trans. 47 (2006) 571-579.

9) S. Farjami, K. Hiraga and H. Kubo: Mater. Trans. 45 (2004) 930-935.

10) S. Farjami and H. Kubo: Mater. Trans. 47 (2006) 564-570.

11) O. Matsumura, T. Sumi, N. Tamura, K. Sakao, T. Furulawa and H. Ohtsuka: Mater. Sci. Eng. A279 (2000) 201-206.

12) J. F. Wan, S. P. Chen, T. Y. Hsu and Y. N. Huang: Mater. Sci. Eng. A 438-440 (2006) 887-890.

13) T. Sawaguchi, P. Sahu, T. Kikuchi, K. Ogawa, S. Kajiwara, A. Kushibe, M. Higashino and T. Ogawa: Scripta Mater. 54 (2006) 1885-1890.

14) N. Bergeon, G. Guenim and C. Esnouf: Mater. Sci. Eng. A242 (1998) 77-86.

15) N. Bergeon, G. Guenim and C. Esnouf: Mater. Sci. Eng. A242 (1998) 87-95.

16) K. Verbeken. N. V. Caenegem and M. Verhaege: Mater. Sci. Eng., in press.

17) H. Fukai, S. Suzuki, N. Masahashi, S. Hanada, T. Maruyama, H. Kubo and Y. Waseda: Mater. Trans. 46 (2005) 1745-1748.

18) B. D. Cullity and S. R. Stock: Elements of X-ray Diffraction, (Prentice Hall, Upper Saddle River, 2001), pp. 407-409.

19) S. Suzuki, Y. Ushigami, H. Homma, S. Takebayashi and T. Kubota: Mater. Trans. 42 (2001) 994-1006.

20) A. Druker, C. Sobrero, H.-G. Brokmeier, J. Malarria and R. Bolmaro: Mater. Sci. Eng. in press. 